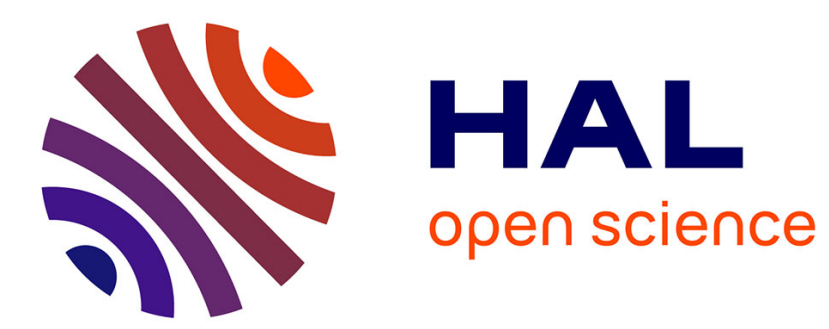

\title{
Basic concepts of representation theory
}

Amritanshu Prasad

\section{To cite this version:}

Amritanshu Prasad. Basic concepts of representation theory. 3rd cycle. Shillong - Inde, 2013, pp.22. cel-00963677

\section{HAL Id: cel-00963677 https://cel.hal.science/cel-00963677}

Submitted on 21 Mar 2014

HAL is a multi-disciplinary open access archive for the deposit and dissemination of scientific research documents, whether they are published or not. The documents may come from teaching and research institutions in France or abroad, or from public or private research centers.
L'archive ouverte pluridisciplinaire HAL, est destinée au dépôt et à la diffusion de documents scientifiques de niveau recherche, publiés ou non, émanant des établissements d'enseignement et de recherche français ou étrangers, des laboratoires publics ou privés. 


\title{
BASIC CONCEPTS OF REPRESENTATION THEORY
}

\author{
AMRITANSHU PRASAD
}

\section{Representations and Modules}

Let $K$ be a field, and $G$ be a finite group. For a $K$-vector space $V$, let $\operatorname{GL}(V)$ denote the group of all invertible $K$-linear maps $V \rightarrow V$.

Definition 1.1 (Representation). A representation of $G$ is a pair $(\rho, V)$, where $V$ is a $K$-vector space and $\rho: G \rightarrow \operatorname{GL}(V)$ is a homomorphism of groups.

Definition 1.2 (Multiplicative character). A multiplicative character of $G$ is a homomorphism $\chi: G \rightarrow K^{*}$.

Each multiplicative character $\chi$ gives rise to a representation as follows: take $V$ to the one dimensional vector space $K$, and take $\rho$ to be the homomorphism which takes $g \in G$ to the linear automorphism of $K$ which multiplies each element by $\chi(g)$.

Exercise 1.3. Show that each multiplicative character of $G$ contains $[G, G]$ in its kernel (and therefore descends to a multiplicative character $G /[G, G] \rightarrow K^{*}$ ). Here $[G, G]$ denotes the subgroup of $G$ generated by elements of the form $x y x^{-1} y^{-1}$ as $x$ and $y$ run over all elements of $G$, and $K^{*}$ denotes the multiplicative group of non-zero elements of $K$.

Representations of groups can be viewed as modules for certain special types of rings which we call $K$-algebras. It is assumed that the reader is familiar with at least the definition of rings, ideals and modules. If not, a quick look at the relevant definitions in a standard textbook (for example, Jacobson [1, Chapter 2]) should suffice.

Definition 1.4 ( $K$-algebra). A $K$-algebra is a ring $R$ whose underlying additive group is a $K$-vector space and whose multiplication operation $R \times R \rightarrow R$ is $K$ bilinear. Only unital $K$-algebras will be considered here, namely those with a multiplicative unit.

Example 1.5. The space $M_{n}(K)$ of $n \times n$ matrices with entries in the field $K$ is a unital $K$-algebra. If $V$ is an $n$-dimensional vector space over $K$, then the choice of a basis for $V$ identifies $M_{n}(K)$ with the algebra $\operatorname{End}_{K} V$ of $K$-linear maps $V \rightarrow V$.

A left ideal of a $K$-algebra $R$ is a linear subspace which is closed under multiplication on the left by elements of $R$. Similarly, a right ideal is a linear subspace which is closed under multiplication on the right by elements of $R$. A two-sided ideal is a subspace which is both a left and a right ideal.

Example 1.6. Let $W \subset K^{n}$ be a linear subspace. Then

$$
\left\{A \in M_{n}(K) \mid A x \in W \text { for all } x \in K^{n}\right\}
$$


is a right ideal in $M_{n}(K)$, while

$$
\left\{A \in M_{n}(K) \mid A x=0 \text { for all } x \in W\right\}
$$

is a left ideal in $M_{n}(K)$.

Exercise 1.7. Show that $M_{n}(K)$ has no two-sided ideals except for the two obvious ones, namely $\{0\}$ and $M_{n}(K)$.

Exercise 1.8. Show that, if $R$ is a $K$-algebra and $I$ is a two-sided ideal in $R$, then the product operation of $R$ descends to a bilinear map $R / I \times R / I \rightarrow R / I$ which makes it a $K$-algebra.

Example 1.9. The polynomial ring $K\left[x_{1}, \ldots, x_{n}\right]$ is a commutative unital $K$ algebra. A large number of $K$-algebras can be constructed by taking quotients of this algebra by its ideals.

A $K$-algebra homomorphism is a homomorphism of rings which is also $K$-linear. It will be assumed that a $K$-algebra homomorphism $R \rightarrow S$ takes the unit of $R$ to the unit of $S$.

The usual definition of modules for a ring can be adapted to $K$-algebras:

Definition 1.10 (Module). For a $K$-algebra $R$, an $R$-module is a pair $(\tilde{\rho}, V)$, where $V$ is a $K$-vector space, and $\tilde{\rho}: R \rightarrow \operatorname{End}_{K} V$ is a $K$-algebra homomorphism.

The notion of an $R$-module in Definition 1.10 requires the $K$-linearity of $\tilde{\rho}$, and is therefore a little stronger than the general definition of a module for a ring (see, for example, Definition 3.1 in Jacobson [1]). But this definition is exactly what is needed to make the correspondence between representations of $G$ and modules of a certain $K$-algebra $K[G]$ associated to $G$, as we shall soon see.

Example 1.11. Every left ideal of $R$ is an $R$-module. Any subspace of an $R$-module $M$ which is closed under the action of $R$ on $M$ can be viewed as an $R$-module in its own right, and is called a submodule. A quotient of an $R$-module by a submodule is also an $R$-module.

Example 1.12. The vector space $K^{n}$ is an $M_{n}(K)$-module when vectors in $K^{n}$ are written as columns and $M_{n}(K)$ acts by matrix multiplication on the left.

The group algebra $K[G]$ of the group $G$ is a $K$-algebra whose additive group is the $K$-vector space with basis

$$
\left\{1_{g} \mid g \in G\right\}
$$

and whose product is defined by bilinearly extending

$$
1_{g} 1_{h}=1_{g h} \text { for all } g, h \in G \text {. }
$$

Another useful way of thinking about the group algebra is as the algebra of $K$-valued functions on $G$ with product given by convolution: if $f_{1}$ and $f_{2}$ are two $K$-valued functions on $G$, their convolution $f_{1} * f_{2}$ is defined by

$$
f_{1} * f_{2}(g)=\sum_{x y=g} f_{1}(x) f_{2}(y) \text { for all } g \in G .
$$

Exercise 1.13. Identify $1_{g}$ with the function whose value at $g$ is 1 , and which vanishes everywhere else. Under this identification, show that the two definitions of the group algebra given above are equivalent. 
Exercise 1.14. Let $n>1$ be an integer. Show that $K[\mathbf{Z} / n \mathbf{Z}]$ is isomorphic to $K[t] /\left(t^{n}-1\right)$ as an algebra. Here $\left(t^{n}-1\right)$ denotes the ideal in $K[t]$ generated by $t^{n}-1$.

If $\rho: G \rightarrow \mathrm{GL}(V)$ is a representation, and one defines a $K$-algebra homomorphism $\tilde{\rho}: K[G] \rightarrow \operatorname{End}_{K}(V)$ by

$$
\tilde{\rho}: f \mapsto \sum_{g \in G} f(g) \rho(g)
$$

for each $f \in K[G]$, then $(\tilde{\rho}, V)$ is a $K[G]$-module.

Conversely, suppose that $\tilde{\rho}: K[G] \rightarrow \operatorname{End}_{K}(V)$ is a $K[G]$-module. Note that if $e$ denotes the identity element of $G$, then $1_{e}$ is the multiplicative unit of $K[G]$. If we assume that $\tilde{\rho}\left(1_{e}\right)=\operatorname{id}_{V}$ (such a module is called unital) then, for any $g \in G$,

$$
\tilde{\rho}\left(1_{g}\right) \tilde{\rho}\left(1_{g^{-1}}\right)=\tilde{\rho}\left(1_{e}\right)=\mathrm{id}_{V},
$$

so $\tilde{\rho}\left(1_{g}\right) \in \mathrm{GL}(V)$. Define a representation $\rho$ of $G$ by

$$
\rho(g)=\tilde{\rho}\left(1_{g}\right) .
$$

The prescriptions (3) and (4) define an equivalence between representations of $G$ and unital $K[G]$-modules. This correspondence makes it possible to use concepts from ring theory in the study of representations of a group.

Example 1.15 (Regular representation). For each $r \in R$, define $\tilde{L}(r)$ to be the linear endomorphism of $R$ obtained by left multiplication by $r$. This turns $R$ into an $R$-module which is known as the left regular $R$-module.

Let us examine the above construction in the case where $R=K[G]$. The group ring $K[G]$ becomes a representation of $G$ if we define $L(g): K[G] \rightarrow K[G]$ by

$$
L(g) 1_{x}=\tilde{L}\left(1_{g}\right) 1_{x}=1_{g x} .
$$

This representation is known as the left regular representation of $G$. If we define $R: G \rightarrow \mathrm{GL}(K[G])$ by

$$
R(g) 1_{x}=1_{x g^{-1}}
$$

we get another representation of $G$ on $K[G]$, which is known as the right regular representation of $G$.

Exercise 1.16. If $K[G]$ is viewed as the space of $K$-valued functions on $G$ (as in Exercise 1.13), then

$$
(L(g) f)(x)=f\left(g^{-1} x\right) \text { and }(R(g) f)(x)=f(x g) .
$$

\section{INVARIANT SUBSPACES AND SIMPLICITY}

Definition 2.1 (Invariant subspace). A subspace $W$ of $V$ is called an invariant subspace for a representation $\rho: G \rightarrow \operatorname{GL}(V)$ if $\rho(g) W \subset W$ for all $g \in G$.

Similarly, a subspace $W$ of $V$ is called an invariant subspace for an $R$-module $\tilde{\rho}: R \rightarrow \operatorname{End}_{K} V$ if $\tilde{\rho}(r) W \subset W$ for all $r \in R$.

Example 2.2. For the left regular representation $(L, K[G])$, the subspace of constant functions is a one dimensional invariant subspace. The subspace

$$
K[G]_{0}=\left\{f: G \rightarrow K \mid \sum_{g \in G} f(g)=0\right\}
$$

is an invariant subspace of dimension $|G|-1$. 
Exercise 2.3. The subspace $K[G]_{0}$ has an invariant complement in $(L, K[G])$ if and only if $|G|$ is not divisible by the characteristic of $K$ (this includes the case where $K$ has characteristic zero).

Exercise 2.4. Let $G=\mathbf{Z} / 2 \mathbf{Z}$ and let $K$ be a field of characteristic two. Show that the subspace of $K[G]$ spanned by $1_{0}+1_{1}$ is the only non-trivial proper invariant subspace for the left (or right) regular representation of $G$.

Exercise 2.5. Show that, if every representation of a group is a sum of one dimensional invariant subspaces, then the group is abelian. Hint: use Exercise 1.3, and the regular representation.

Definition 2.6 (Simplicity). A representation or module is said to be simple (or irreducible) if it has no non-trivial proper invariant subspaces. As a convention, the representation or module with a zero dimensional vector space is not considered to be simple.

Example 2.7. Every one dimensional representation is simple.

Exercise 2.8. Every simple module for a finite dimensional $K$-algebra is finite dimensional.

Exercise 2.9. If $K$ is algebraically closed, and $G$ is abelian, then every simple representation of $G$ is of dimension one. Hint: Show that, for any commuting family of matrices in an algebraically closed field, there is a basis with respect to which all the matrices in that family are upper triangular.

Example 2.10. The hypothesis that $K$ is algebraically closed is necessary in Exercise 2.9. Take for example, $G=\mathbf{Z} / 4 \mathbf{Z}$, and $\rho: G \rightarrow G L_{2}(\mathbf{R})$ the representation which takes a generator of $\mathbf{Z} / 4 \mathbf{Z}$ to the matrix $\left(\begin{array}{cc}0 & 1 \\ -1 & 0\end{array}\right)$. Since this matrix is a rotation by $\pi / 2$, no line in $\mathbf{R}^{2}$ is left invariant by it, and so the abelian group $\mathbf{Z} / 4 \mathbf{Z}$ admits a simple two dimensional representation over the real numbers.

Definition 2.11 (Intertwiners). Let $\left(\rho_{1}, V_{1}\right)$ and $\left(\rho_{2}, V_{2}\right)$ be representations of $G$. A linear transformation $T: V_{1} \rightarrow V_{2}$ is called an intertwiner (or a $G$-homomorphism) if

$$
T \circ \rho_{1}(g)=\rho_{2}(g) \circ T \text { for all } g \in G .
$$

The space of all intertwiners $V_{1} \rightarrow V_{2}$ is denoted $\operatorname{Hom}_{G}\left(V_{1}, V_{2}\right)$.

Similarly, for $R$-modules $\left(\tilde{\rho}_{1}, V_{1}\right)$ and $\left(\tilde{\rho}_{2}, V_{2}\right)$ an intertwiner is a linear transformation $T: V_{1} \rightarrow V_{2}$ such that

$$
T \circ \tilde{\rho}_{1}(r)=\tilde{\rho}_{2}(r) \circ T \text { for all } r \in R \text {. }
$$

The space of all such intertwiners is denoted by $\operatorname{Hom}_{R}\left(V_{1}, V_{2}\right)$.

The intertwiner condition (5) can be visualized as a commutative diagram:

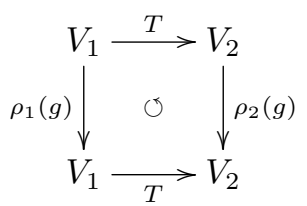


If one begins with an element in the top-right corner of this diagram, the images obtained by applying the functions along either of the two paths to the bottom-right corner are the same.

Exercise 2.12. The kernel of an intertwiner is an invariant subspace of its domain and the image is an invariant subspace of its codomain.

Theorem 2.13 (Schur's lemma I). If $K$ is algebraically closed and $V$ is a finite dimensional simple representation of $G$ then every self-intertwiner $T: V \rightarrow$ $V$ is a scalar multiple of the identity map. In other words, $\operatorname{End}_{G} V=K \mathrm{id}_{V}$ $\left(\operatorname{End}_{G} V\right.$ denotes $\operatorname{Hom}_{G}(V, V)$, the self-intertwiners of $V$, which are also called $G$-endomorphisms of $V$ ).

Proof. Since $K$ is algebraically closed, any self-intertwiner $T: V \rightarrow V$ has an eigenvalue, say $\lambda$. Now $T-\lambda \mathrm{id}_{V}$ is also an intertwiner. Moreover, it has a nontrivial kernel. Since its kernel is an invariant subspace (Exercise 2.12), it must (by the simplicity of $V$ ) be all of $V$. Therefore $T=\lambda \mathrm{id}_{V}$.

A similar statement (with the same proof) holds for simple modules of a $K$ algebra.

Exercise 2.14 (Central character). When $K$ is algebraically closed, show that the centre $Z(G)$ of $G$ acts on any simple representation by scalar matrices (if $g \in Z(G)$ acts by the scalar matrix $\lambda(g) I$, then $g \mapsto \lambda(g)$ is a homomorphism $Z(G) \rightarrow K^{*}$, which is called the central character of the representation).

Exercise 2.15 (Schur's lemma for arbitrary fields). Let $K$ be any field (not necessarily algebraically closed). Show that any non-zero self-intertwiner of a simple representation (or module) is invertible.

Definition 2.16 (Isomorphism). We say that representations (or modules) $V_{1}$ and $V_{2}$ are isomorphic (and write $V_{1} \cong V_{2}$ or $\rho_{1} \cong \rho_{2}$ ) if there exists an invertible intertwiner $V_{1} \rightarrow V_{2}$ (its inverse will be an intertwiner $V_{2} \rightarrow V_{1}$ ).

Exercise 2.17. Near the beginning of Section 1 we saw that multiplicative characters give rise to one dimensional representations. Show that this constuction gives rise to a bijection from the set of multiplicative characters of a finite group $G$ to its set of isomorphism classes of one dimensional representations.

Theorem 2.18 (Schur's lemma II). If $V_{1}$ and $V_{2}$ are simple, then every non-zero intertwiner $T: V_{1} \rightarrow V_{2}$ is an isomorphism. Consequently, either $V_{1} \cong V_{2}$ or there are no non-zero intertwiners $V_{1} \rightarrow V_{2}$.

Proof. If $T$ is a non-zero intertwiner then its kernel is an invariant subspace of $V_{1}$. Since this kernel can not be all of $V_{1}$, it is trivial, hence $T$ is injective. Its image, being a non-trivial invariant subspace of $V_{2}$ must be all of $V_{2}$, therefore $T$ is an isomorphism.

An easy consequence of the two previous results is:

Corollary 2.19. If $K$ is algebraically closed, $V_{1}$ and $V_{2}$ are simple and $T: V_{1} \rightarrow V_{2}$ is any non-trivial intertwiner, then $\operatorname{Hom}_{G}\left(V_{1}, V_{2}\right)=K T$.

Proof. $T$ is invertible by Schur's Lemma II. If $S: V_{1} \rightarrow V_{2}$ is another intertwiner, then $T^{-1} \circ S$ is a self-intertwiner of $V_{1}$. By Schur's Lemma I, $T^{-1} S=\lambda \mathrm{id}_{V_{1}}$ for some $\lambda \in K$, whence $S=\lambda T$.

Endomorphisms of simple modules are all multiples of the identity.

Isomorphism classes of one dimensional representations

are multiplicative characters.

Homomorphisms between simple modules form a vector space of dimension at most one. 


\section{Complete Reducibility}

Definition 3.1 (Completely Reducible Module). An $R$-module is said to be completely reducible if it is a direct sum of simple modules.

We have already seen (Exercises 2.3 and 2.4) that not all modules are completely reducible.

Exercise 3.2. Show that an $R$-module is completely reducible if and only if every invariant subspace has an invariant complement.

Exercise 3.3. Show that an invariant subspace of a completely reducible module is completely reducible. Also, the quotient of a completely reducible module by any invariant subspace is completely reducible.

Exercise 3.4. If the left regular $R$-module is completely reducible, then every $R$-module is completely reducible.

If $V$ is a finite dimensional completely reducible $R$-module, then

$$
V \cong V_{1}^{\oplus m_{1}} \oplus V_{2}^{\oplus m_{2}} \oplus \cdots \oplus V_{r}^{\oplus m_{r}}
$$

where (by grouping the simple subspaces of $V$ which are isomorphic together) $V_{1}, V_{2}, \ldots, V_{r}$ is a collection of pairwise non-isomorphic simple $R$-modules. The number $m_{k}$ is called the multiplicity of $V_{k}$ in $V$. We shall refer to (6) as the decomposition of $V$ into simple modules with multiplicities. Let $W$ be another finite dimensional completely reducible module whose decomposition into simple modules with multiplicities is

$$
W \cong V_{1}^{\oplus n_{1}} \oplus V_{2}^{\oplus n_{2}} \oplus \cdots V_{r}^{\oplus n_{r}}
$$

(by allowing some of the $n_{k}$ 's and $m_{k}$ 's to be 0 , we may assume that the underlying collection $V_{1}, V_{2}, \ldots, V_{r}$ of simple modules is the same for $V$ and $W$ ). Since there are no intertwiners $V_{i} \rightarrow V_{j}$ for $i \neq j$, any $T \in \operatorname{Hom}_{R}(W, V)$ can be expressed as

$$
T=\bigoplus_{k} T_{k}
$$

where $T_{k}: V_{k}^{\oplus n_{k}} \rightarrow V_{k}^{\oplus m_{k}}$ is an intertwiner. Represent an element $x \in V_{k}^{\oplus n_{k}}$ as a vector $\left(x_{1}, \ldots, x_{n_{k}}\right)$ and $y \in V_{k}^{\oplus m_{k}}$ as $y=\left(y_{1}, \ldots, y_{m_{k}}\right)$, with each $x_{i}, y_{i} \in V_{k}$. Writing these vectors as columns, the intertwiner $T_{k}$ can itself be expressed as an $m_{k} \times n_{k}$ matrix $T_{k}=\left(T_{i j}\right)$ (where $\left.T_{i j} \in \operatorname{End}_{R} V_{k}\right)$ using

$$
\left(\begin{array}{c}
T(x)_{1} \\
T(x)_{2} \\
\vdots \\
T(x)_{m_{k}}
\end{array}\right)=\left(\begin{array}{cccc}
T_{11} & T_{12} & \cdots & T_{1 n_{k}} \\
T_{21} & T_{22} & \cdots & T_{2 n_{k}} \\
\vdots & \vdots & \ddots & \vdots \\
T_{m_{k} 1} & T_{m_{k} 2} & \cdots & T_{m_{k} n_{k}}
\end{array}\right)\left(\begin{array}{c}
x_{1} \\
x_{2} \\
\vdots \\
x_{n_{k}}
\end{array}\right) .
$$

Homomorphisms between modules made up of copies of the same simple module can be thought of as matrices with entries in $K$.
If $K$ is algebraically closed, then Schur's lemma I (Theorem 2.13) holds. Thus, the entries of the matrix, being scalar multiples of the identity $\operatorname{id}_{V_{k}}$ can themselves be thought of as scalars, allowing us to write

$$
\operatorname{Hom}_{R}(W, V)=\bigoplus_{k=1}^{r} M_{m_{k} \times n_{k}}(K)
$$

where $M_{m_{k} \times n_{k}}$ denotes the set of $m_{k} \times n_{k}$ matrices with entries in $K$. One easily checks that composition of intertwiners expressed as matrices in the above manner corresponds to multiplication of matrices. 
Theorem 3.5. If $K$ is algebraically closed and $V$ and $W$ have decompositions into sums of simple modules with multiplicities given by (6) and (7), then

$$
\operatorname{dim} \operatorname{Hom}_{R}(V, W)=\operatorname{dim} \operatorname{Hom}_{R}(W, V)=\sum_{i} m_{i} n_{i} .
$$

In the special case where $W=V$, we obtain

Theorem 3.6. Let $K$ be an algebraically closed field and $R$ be a $K$-algebra. If the $R$-module $V$ is a sum of non-isomorphic simple modules with multiplicities given by (6), then $\operatorname{End}_{R} V$ is a sum of matrix algebras (with componentwise multiplication):

$$
\operatorname{End}_{R} V \cong \bigoplus_{i=1}^{r} M_{m_{i}}(K)
$$

The Endomorphism algebra of a completely reducible module is a sum of matrix algebras.

where the right hand side should be interpreted as a sum of algebras.

\section{Sum of algebras}

The notion of a sum of algebras will come up often and therefore deserves a short discussion.

Definition 3.7 (Sum of algebras). If $R_{1}, R_{2}, \ldots, R_{k}$ are algebras, their sum is the algebra whose underlying vector space is the direct sum $R:=R_{1} \oplus R_{2} \oplus$ $\cdots \oplus R_{k}$, with multiplication defined componentwise:

$$
\left(r_{1}+r_{2}+\cdots+r_{k}\right)\left(s_{1}+s_{2}+\cdots+s_{k}\right)=r_{1} s_{1}+r_{2} s_{2}+\cdots+r_{k} s_{k}
$$

Thus each $R_{i}$ is a subalgebra of $R$ for each $i$. If each of the algebras $R_{i}$ is unital with unit $1_{i}$, then the sum

$$
1:=1_{1}+1_{2}+\cdots+1_{k}
$$

is the multiplicative unit for $R$. In particular, $R$ is also unital. If $\left(\tilde{\rho}_{i}, M_{i}\right)$ is a unital $R_{i}$ module (meaning that $\tilde{\rho}_{i}\left(1_{i}\right)=\mathrm{id}_{M_{i}}$ ) then

$$
M=M_{1} \oplus M_{2} \oplus \cdots \oplus M_{k}
$$

is also a unital $R$-module when $\tilde{\rho}: R \rightarrow \operatorname{End}_{K} M$ is defined by

$$
\tilde{\rho}\left(r_{1}+r_{2}+\cdots+r_{k}\right):=\tilde{\rho}_{1}\left(r_{1}\right)+\tilde{\rho}_{2}\left(r_{2}\right)+\cdots+\tilde{\rho}_{k}\left(r_{k}\right) .
$$

The $M_{i}$ 's can be recovered from $M$ by $M_{i}=\tilde{\rho}\left(1_{i}\right) M$. Thus $R$-modules correspond precisely to collections of $R_{i}$-modules (one for each $i$ ).

On a purely combinatorial level:

Theorem 3.8. Assume that $K$ is algebraically closed. If the $R$-module $V$ is a sum of non-isomorphic simple modules with multiplicities given by (6), then

$$
\operatorname{dim} \operatorname{End}_{R} V=\sum_{i=1}^{r} m_{i}^{2} .
$$

Recall that the centre of a $K$-algebra $R$ consists of those elements which commute with every element of $R$.

We all know that the centre of a matrix algebra consists of the scalar matrices. The centre of a direct sum of algebras is the direct sum of their centres. It follows that the dimension of the centre of $\bigoplus_{i=1}^{r} M_{m_{i}}(K)$ is the number of $i$ such that $m_{i}>0$. Thus a consequence of Theorem 3.6 is 
Theorem 3.9. Let $R$ be a $K$-algebra, with $K$ algebraically closed. If the $R$-module $V$ is a sum of non-isomorphic simple modules with multiplicities given by (6) with all the multiplicities $m_{i}>0$, then the dimension of the centre of $\operatorname{End}_{R} V$ is $r$.

Exercise 3.10. Let $R$ be a $K$-algebra, where $K$ is an algebraically closed field. Show that a completely reducible $R$-module $V$ is simple if and only if $\operatorname{dim}_{\operatorname{End}_{R}} V=$ 1.

Exercise 3.11. Assume that $K$ is algebraically closed, that $V$ is simple and that $W$ is completely reducible. Then $\operatorname{dim} \operatorname{Hom}_{R}(V, W)$ is the multiplicity of $V$ in $W$.

A completely reducible module has a multiplicity-free decomposition if and only its endomorphism algebra is commutative.

A completely reducible module with a multiplicity-free decomposition into finitely many simple modules has only finitely many invariant subspaces.
Exercise 3.12. Assume that $K$ is algebraically closed. A completely reducible $R$-module $V$ has a multiplicity-free decomposition (meaning that its decomposition into simple modules with multiplicities is of the form (6) with $m_{i}=1$ for all $i$ ) if and only if its endomorphism algebra $\operatorname{End}_{R} V$ is commutative.

Exercise 3.13. Assume that $K$ is algebraically closed. If $V$ and $W$ are completely reducible finite dimensional $R$-modules such that

$$
\operatorname{dim} \operatorname{End}_{R} V=\operatorname{dim} \operatorname{Hom}_{R}(V, W)=\operatorname{dim} \operatorname{End}_{R} W
$$

then $V$ and $W$ are isomorphic.

Exercise 3.14. Two $R$-modules $V$ and $W$, satisfying (6) and (7), where $V_{1}, V_{2}, \ldots, V_{r}$ are pairwise non-isomorphic simple $R$-modules, are isomorphic if and only if $m_{i}=n_{i}$ for $i=1,2, \ldots, r$.

Exercise 3.15. Assume that $K$ is algebraically closed. Suppose that $V_{1}, \ldots, V_{r}$ are pairwise non-isomorphic simple $R$-modules. Show that every invariant subspace of $V=V_{1} \oplus \cdots \oplus V_{r}$ is of the form $V_{i_{1}} \oplus \cdots \oplus V_{i_{k}}$ for some $1 \leq i_{1}<\cdots<i_{k} \leq r$. In contrast, if $n \geq 2$, then $V^{\oplus n}$ has infinitely many invariant subspaces if $K$ is infinite. Hint: using Exercise 3.11, $\operatorname{Hom}_{R}\left(V_{i}, V\right)$ is of dimension one.

\section{MASCHKE'S THEOREM}

In Exercise 2.3 we saw that when the characteristic of $K$ divides $|G|$ then an invariant subspace of a representation need not contain a complement. On the other hand, we have

Theorem 4.1 (Maschke). If $(\rho, V)$ is a representation of $G$ and the characteristic of the field $K$ does not divide $|G|$, then every invariant subspace of $V$ has an invariant complement.

Proof of Maschke's theorem. Let $W$ be an invariant subspace and let $U$ be any complement (not necessarily invariant) of $W$. Thus $V=W \oplus U$, meaning that every vector $x \in V$ can be uniquely written in the form $x=x_{W}+x_{U}$. Define projection operators on $V$ by

$$
P_{W}(x)=x_{W} \text { and } P_{U}(x)=x_{U} \text { for all } x \in V .
$$

Note that $P_{W}$ depends not just on $W$, but also on $U$.

Maschke's theorem is proved by constructing an invariant complement from the arbitrary complement $U$ by averaging projection operators. The following lemma gives an interpretation of the invariance of $U$ in terms of the projection operators $P_{W}$ and $P_{U}$ : 
Lemma 4.2. The subspace $U$ is also invariant for $\rho$ if and only if $P_{W} \in \operatorname{End}_{G} V$ (equivalently, if and only if $P_{U} \in \operatorname{End}_{G} V$, since $P_{W}+P_{U}=\operatorname{id}_{V}$ ).

Proof of lemma. Since $P_{W}\left(x_{W}\right)=x_{W}$, we have

$$
\rho(g) P_{W}\left(x_{W}\right)=\rho(g) x_{W} .
$$

Since $W$ is invariant, $\rho(g) x_{W} \in W$ and therefore,

$$
P_{W}\left(\rho(g)\left(x_{W}\right)\right)=\rho(g) x_{W} .
$$

It follows that

$$
P_{W} \circ \rho(g)\left(x_{W}\right)=\rho(g) \circ P_{W}\left(x_{W}\right) \text { for all } g \in G .
$$

On the other hand, since $P_{W}\left(x_{U}\right)=0$,

$$
\rho(g)\left(P_{W}\left(x_{U}\right)\right)=0 .
$$

But $P_{W}\left(\rho(g) x_{U}\right)=0$ if and only if $\rho(g) x_{U} \in U$. It follows that the identity

$$
P_{W}\left(\rho(g) x_{U}\right)=\rho(g)\left(P_{W}\left(x_{U}\right)\right) \text { for all } g \in G
$$

holds for all $x_{U} \in U$ if and only if $U$ is an invariant subspace. Therefore the intertwining property, namely that

$$
\rho(g) \circ P_{W}=P_{W} \circ \rho(g) \text { for all } g \in G
$$

being the sum of (8) and (9), holds if and only if $U$ is an invariant subspace. This completes the proof of the lemma.

In order to complete the proof of Maschke's theorem, define

$$
\overline{P_{W}}=\frac{1}{|G|} \sum_{g \in G} \rho(g) P_{W} \rho(g)^{-1} .
$$

Note that the hypothesis that $|G|$ is not divisible by the characteristic is necessary in order to make sense of division by $|G|$. It is easy to see that $\overline{P_{W}}$ is a self-intertwiner:

$$
\rho(g) \overline{P_{W}}=\overline{P_{W}} \rho(g) \text { for all } g \in G .
$$

If $x \in W$ then $\rho(g)^{-1}(x) \in W$. Therefore $P_{W}\left(\rho(g)^{-1}(x)\right)=\rho(g)^{-1}(x)$. It follows that $\rho(g) \overline{P_{W}} \rho(g)^{-1}(x)=x$, from which one concludes that for $x \in W, \overline{P_{W}}(x)=x$. Now, if $x \in V$, then $\overline{P_{W}}(x) \in W$ and therefore, $\overline{P_{W}}\left(\overline{P_{W}}(x)\right)=\overline{P_{W}}(x)$. Thus

$$
{\overline{P_{W}}}^{2}=\overline{P_{W}} .
$$

Let $\overline{P_{U}}=\mathrm{id}_{V}-\overline{P_{W}}$. One easily checks that ${\overline{P_{U}}}^{2}=\overline{P_{U}}$. Let $\bar{U}$ denote the image of $\overline{P_{U}}$. Every vector $x \in V$ can be written as

$$
x=\overline{P_{W}}(x)+\overline{P_{U}}(x),
$$

so $V=W+\bar{U}$.

We have seen that if $x \in W$, then $\bar{P}_{W}(x)=x$. Therefore

$$
\overline{P_{U}}(x)=\left(1-\overline{P_{W}}\right)(x)=0 \text { for all } x \in W .
$$

On the other hand, if $x \in \bar{U}, x=\overline{P_{U}}\left(x^{\prime}\right)$ for some $x^{\prime} \in V$. Using the fact that ${\overline{P_{U}}}^{2}=\overline{P_{U}}$,

$$
\overline{P_{U}}(x)={\overline{P_{U}}}^{2}\left(x^{\prime}\right)=\overline{P_{U}}\left(x^{\prime}\right)=x \text { for all } x \in \bar{U} .
$$


Together, (10) and (11) imply that $W \cap \bar{U}=0$. Therefore $V=W \oplus \bar{U}$ (with corresponding projection operators $\overline{P_{W}}$ and $\overline{P_{U}}$ ). Thus, by Lemma $4.2, \bar{U}$ is an invariant complement of $W$.

Theorem 4.3 (Complete Reducibility of representations). If $(\rho, V)$ is a finite dimensional representation of $G$ and the characteristic of $K$ does not divide $|G|$, then $V$ is completely reducible.

Proof. Easily follows from Maschke's theorem (Theorem 4.1).

Exercise 4.4. Let $A$ be a matrix with entries in an algebraically closed field $K$. Suppose that $A^{n}=1$ for some $n$ not divisible by the characteristic of $K$. Use Maschke's theorem and Exercise 2.9 to show that $A$ is diagonalizable.

Definition 4.5 (Semisimple algebra). A finite dimensional $K$-algebra $R$ is said to be semisimple if every finite dimensional $R$-module $M$ is a sum of simple invariant

Maschke's theorem tells us when $K[G]$ is a semisimple algebra.

All simple modules are submodules of the regular module. subspaces.

Maschke's theorem tells us that if the characteristic of $K$ does not divide $|G|$ then $K[G]$ is a semisimple algebra. The converse also holds: if the characteristic of $K$ divides $|G|$, then $K[G]$ is not semisimple since the regular module admits an invariant subspace that does not have an invariant complement (see Exercise 2.3).

The following exercise illustrates another application of the techniques used in the proof of Maschke's theorem.

Exercise 4.6. Let $N$ be a normal subgroup of $G$. If $V$ is a simple representation of $G$, then its restriction to $N$ is completely reducible.

Exercise 4.7. Consider a representation $V$ of $G$ in a vector space over C. Show that $V$ admits a $G$-invariant Hermitian inner product, namely an Hermitian inner product $\langle\cdot, \cdot\rangle$ such that

$$
\langle g \cdot x, g \cdot y\rangle=\langle x, y\rangle \text { for all } g \in G, x, y \in V .
$$

Hint: Start with any Hermitian form on $V$ and average it out over $G$.

Exercise 4.8. Use Exercise 4.7 to give another proof of Maschke's theorem for representations over $\mathbf{C}$.

\section{Decomposing the Regular module}

In view of complete reducibility, classifying the simple modules up to isomorphism is equivalent to classifying all the finite dimensional modules up to isomorphism.

In order to find simple modules, we do not have to look beyond the regular module (namely $R$ itself, thought of as an $R$-module; see Example 1.15):

Theorem 5.1. If $R$ is semisimple then every simple $R$-module is isomorphic to a submodule of the left regular $R$-module.

Proof. Given a simple $R$-module $V$, choose a non-zero vector $x \in V$. Define $\phi_{x}$ : $R \rightarrow V$ by

$$
\phi_{x}(r)=r x \text { for all } r \in R .
$$

Then $\phi_{x}$ is a non-zero intertwiner from the left regular $R$-module to $V$. It follows from Theorem 3.5 that $V$ is isomorphic to submodule of $R$, as claimed. 
Notice that Theorem 5.1 also shows that, up to isomorphism, there are only finitely many simple $R$-modules, namely, those which are isomorphic to some simple invariant subspace of the regular $R$-module.

Indeed, if we express the left-regular $R$-module in the form

$$
R=V_{1}^{\oplus m_{1}} \oplus V_{2}^{\oplus m_{2}} \oplus \cdots \oplus V_{r}^{\oplus m_{r}}
$$

with $V_{1}, V_{2}, \ldots, V_{r}$ pairwise non-isomorphic, then any simple $R$-module will be isomorphic to exactly one of $V_{1}, V_{2}, \ldots, V_{r}$.

By Theorem 3.6, if (12) holds, then

$$
\operatorname{End}_{R} R \cong \bigoplus_{i=1}^{r} M_{m_{i}}(K) .
$$

Moreover, it is not difficult to relate $\operatorname{End}_{R} R$ to the $K$-algebra $R$ :

Exercise 5.2. Let $R$ be a $K$-algebra with a multiplicative unit. For each $r \in R$, define $\psi_{r}: R \rightarrow R$ by

$$
\psi_{r}(s)=s r \text { for all } s \in R
$$

Show that $r \mapsto \psi_{r}$ is a vector-space isomorphism $R \rightarrow \operatorname{End}_{R} R$. [Hint: for surjectivity, note that $r$ can be recovered from $\psi_{r}$ as $\left.\psi_{r}(1)\right]$.

Both $R$ and $\operatorname{End}_{R} R$ are $K$-algebras. How does the isomorphism of Exercise 5.2 relate these algebra structures? We calculate:

$$
\begin{aligned}
\psi_{r} \circ \psi_{r^{\prime}}(s) & =\psi_{r^{\prime}}(s) r \\
& =\left(s r^{\prime}\right) r \\
& =\psi_{r^{\prime} r}(s) .
\end{aligned}
$$

Thus $r \mapsto \psi_{r}$ is not an algebra homomorphism; rather it reverses the order of multiplication.

Reversing the order of multiplication in a $K$-algebra results in another $K$-algebra.

Definition 5.3 (Opposite algebra). If $R$ is a $K$-algebra, then its opposite algebra $R^{\text {opp }}$ is the $K$-algebra whose underlying vector space is $R$, but whose multiplication operation is given by reversing the order of multiplication in $R$ :

$$
r s\left(\text { product in } R^{\text {opp }}\right)=s r(\text { product in } R) \text {. }
$$

We can now state the exact relationship between the algebras $\operatorname{End}_{R} R$ and $R$ :

Theorem 5.4. For any $K$-algebra $R$, the map $r \mapsto \psi_{r}$ defined in (13) is an isomorphism of algebras $R^{\mathrm{opp}} \rightarrow \operatorname{End}_{R} R$.

There are many non-commutative algebras which are isomorphic to their opposite algebras:

Exercise 5.5. An isomorphism of $K$-algebras $M_{n}(K) \rightarrow M_{n}(K)^{\text {opp }}$ is given by $T \mapsto T^{t}$.

Exercise 5.6. Let $G$ be a group. An isomorphism of $K$-algebras $K[G] \rightarrow K[G]^{\text {opp }}$ is defined by $1_{g} \mapsto 1_{g^{-1}}$ for each $g \in G$.

Matrix algebras and group algebras are isomorphic to their opposites. 
Every semisimple algebra is a sum of matrix algebras.

All simple modules for a matrix algebra are isomorphic.
Exercise 5.7. The $K$-algebra of upper triangular $n \times n$ matrices is isomorphic to the opposite of the $K$-algebra of lower triangular $n \times n$ matrices. The $K$-algebra of upper triangular $n \times n$ matrices is also isomorphic to the $K$-algebra of lower triangular $n \times n$ matrices. Thus the $K$-algebra of upper triangular $n \times n$ matrices is isomorphic to its own opposite.

Can you think of an algebra that is not isomorphic to its opposite?

Returning to our semisimple algebra $R$, we find that $R$ is isomorphic to $\left(\operatorname{End}_{R} R\right)^{\text {opp }}$, which in turn is isomorphic to a sum of matrix algebras, which are isomorphic to their own opposites. We get:

Theorem 5.8 (Wedderburn decomposition). When $K$ is an algebraically closed field, every semisimple $K$-algebra is isomorphic to a sum of matrix algebras.

Thus matrix algebras are the building blocks of semisimple algebras. If our goal is to understand modules for semisimple algebras, it makes sense to first understand modules for matrix algebras (see the dicussion accompanying Definition 3.7). To begin with, let us first understand the invariant subspaces in the left regular $R$ module when $R$ is the matrix algebra $M_{n}(K)$.

Exercise 5.9. For each linear subspace $V$ of $K^{n}$ define $M_{V}$ to be the set of all matrices whose rows, when thought of as elements of $K^{n}$, lie in $V$.

(1) $M_{V}$ is an invariant subspace of the left regular $M_{n}(K)$-module of dimension $n \operatorname{dim}_{K} V$.

(2) Every invariant subspace of the left regular $M_{n}(K)$-module is of the form $M_{V}$ for some linear subspace $V$ of $K^{n}$.

(3) $M_{V}$ is simple if and only if $V$ is one dimensional.

(4) $M_{V}$ is isomorphic to $M_{W}$ as an $M_{n}(K)$-module if and only if the two subspaces $V$ and $W$ have the same dimension.

For example, take $U_{i}$ to be the line spanned by the $i$ th coordinate vector. Then $M_{U_{i}}$ consists of all matrices whose non-zero entries all lie in the $i$ th column. By Exercise 5.9, the $M_{U_{i}}$ 's are simple invariant subspaces which are isomorphic to each other

$$
M_{n}(K)=M_{U_{1}} \oplus M_{U_{2}} \oplus \cdots \oplus M_{U_{n}} \cong M_{U_{1}}^{\oplus n}
$$

is the decomposition of the left regular $M_{n}(K)$-module into simple invariant subspaces.

The vector space $K^{n}$ is itself a $M_{n}(K)$-module when $M_{n}(K)$ acts by left multiplication on column vectors. Define a linear map $K^{n} \rightarrow M_{U_{i}}$ by taking $x \in K^{n}$ to the matrix whose $i$ th column is $x$ and all other columns are 0 . This is an isomorphism $K^{n} \rightarrow M_{U_{i}}$ of $M_{n}(K)$-modules.

We obtain the decomposition of the left regular $M_{n}(K)$-module:

Theorem 5.10. As an $M_{n}(K)$-module

$$
M_{n}(K) \cong K^{n} \oplus K^{n} \oplus \cdots \oplus K^{n} \text { (n times). }
$$

By Theorem 5.1, every simple module for $M_{n}(K)$ is isomorphic to the module $K^{n}$.

From Theorem 5.10, we can get the decomposition of the regular module for any sum of matrix algebras: 
Corollary 5.11. Let

$$
R=\bigoplus_{i=1}^{r} M_{m_{i}}(K) .
$$

Let $\left(\tilde{\rho}_{i}, V_{i}\right)$ denote the $R$-module whose underlying vector space $V_{i}$ is $K^{m_{i}}$, regarded as column vectors and if

$$
\left.r=r_{1}+r_{2}+\cdots+r_{k} \quad r_{j} \in M_{m_{j}}(K)\right),
$$

then $\tilde{\rho}_{i}(r)(x)=r_{i} x$ for $x \in V_{i}$ (left multiplication of a column vector by a matrix). The decomposition of the left regular $R$-module into simples is given by

$$
R \cong \bigoplus_{i=1}^{r} V_{i}^{\oplus m_{i}}
$$

Corollary 5.11 can be rephrased as follows:

Corollary 5.12. If $K$ is an algebraically closed field and $R$ is a semisimple $K$ algebra, then there is an isomorphism of algebras

$$
R \cong \bigoplus_{\left(\tilde{\rho}_{i}, V_{i}\right)} \operatorname{End}_{K} V_{i}
$$

where $\left(\tilde{\rho}_{i}, V_{i}\right)$ runs over the set of isomorphism classes of simple $R$-modules.

The following exercise should convince the reader that the Wedderburn decomposition is unique:

Exercise 5.13. An element $\epsilon \in R$ is said to be idempotent if $\epsilon^{2}=\epsilon$. A central idempotent is an idempotent that lies in the centre of $R$ (i.e., it commutes with every $r \in R$ ). A central idempotent is said to be primitive if it can not be written as $\epsilon_{1}+\epsilon_{2}$ where $\epsilon_{1}$ and $\epsilon_{2}$ are both non-zero central idempotents.

In the ring $R=\bigoplus_{i=1}^{k} M_{m_{i}}(K)$, let $\epsilon_{i}$ denote the identity matrix of the $i$ th summand. Show that $\epsilon_{1}, \epsilon_{2}, \ldots, \epsilon_{k}$ are all the primitive central idempotents of $R$. The $i$ th matrix algebra can be isolated in $R$ as the two-sided ideal $R \epsilon_{i} R$. Clearly, the multiplicative unit 1 of $R$ is the sum of the primitive central idempotents:

$$
1=\epsilon_{1}+\epsilon_{2}+\cdots+\epsilon_{k} .
$$

Corollary 5.14. Let $G$ be a finite group and $K$ be an algebraically closed field whose characteristic does not divide $|G|$. Let $\left(\rho_{i}, V_{i}\right), i=1, \ldots, r$ be a complete set of representatives of the isomorphism classes of simple representations of $G$. Let $d_{i}=\operatorname{dim} V_{i}$. Then

(1) $d_{1}^{2}+\ldots+d_{r}^{2}=|G|$.

(2) $r$ is the number of conjugacy classes of $G$.

Proof. By Corollary 5.12 and the equivalence between $K[G]$-modules and representations of $G$ discussed in Section 1,

$$
K[G]=\bigoplus_{i=1}^{r} \operatorname{End}_{K}\left(V_{i}\right) .
$$

Comparing the dimensions of the two sides of the above isomorphism gives (1).

Since $r$ is the dimension of the centre of $\bigoplus_{i=1}^{r} M_{m_{i}}(K)$ which is isomorphic to $K[G]$, it suffices to show that the centre of $K[G]$ has dimension equal to the number

The Wedderburn decomposition can be recovered from the primitive central idempotents. 
of conjugacy classes in $G$. For $\sum_{g} a_{g} 1_{g}$ to lie in the centre of $K[G]$, it suffices that it commute with $1_{x}$ for each $x \in G$, i.e.,

$$
\sum_{g} a_{g} 1_{x g}=\sum_{g} a_{g} 1_{g x} \text { for each } x \in G .
$$

By re-indexing the sums, we may write

$$
\sum_{g} a_{x^{-1} g} 1_{g}=\sum_{g} a_{g x^{-1}} 1_{g} \text { for each } x \in G .
$$

Comparing the coefficients of $1_{g}$ on both sides of the above equality gives

$$
a_{x^{-1} g}=a_{g x^{-1}} \text { for all } x, g \in G .
$$

Replacing $g$ by $g x$ in the above identity gives

$$
a_{x^{-1} g x}=a_{g} \text { for all } x, g \in G .
$$

Thus $\sum_{g} a_{g} 1_{g}$ is in the centre of $K[G]$ if and only if the function $g \mapsto a_{g}$ is constant on the conjugacy classes of $G$. It follows that the dimension of the centre of $K[G]$ is the number of conjugacy classes of $G$, completing the proof of (2).

If $(\tilde{\rho}, V)$ is an $R$-module for a finite dimensional $K$-algebra $R$, and $\tilde{\rho}(R)=$ $\operatorname{End}_{K} V$, then clearly, $V$ can not admit a non-trivial proper invariant subspace. Therefore $V$ is simple. The converse is true whenever $K$ is algebraically closed. When $R$ is semisimple, then this is easier to prove and goes by the name of Burnside's theorem.

Theorem 5.15 (Burnside's theorem). Let $K$ be an algebraically closed field and $R$ be a finite dimensional semisimple $K$-algebra. If $(\tilde{\rho}, V)$ is a simple $R$-module then $\tilde{\rho}(R)=\operatorname{End}_{K} V$.

Proof. Think of $\operatorname{End}_{K} V$ as an $R$-module using $r \cdot T=\tilde{\rho}(r) \circ T$. Let $x_{1}, \ldots, x_{n}$ be a basis of $V$ over $K$. If we write $T \in \operatorname{End}_{K} V$ as a matrix with respect to this basis, then each column of this matrix can be thought of as a vector in $V$, and the action of $R$ on $\operatorname{End}_{K} V$ is coincides with the action of $R$ on each column under this identification. Therefore, as $R$-modules,

$$
\operatorname{End}_{K} V \cong V^{\oplus n} .
$$

Now the image $\bar{R}$ of $R$ in $\operatorname{End}_{K} V$, being a subalgebra of $\operatorname{End}_{K} V$, can be viewed as a submodule of $\operatorname{End}_{K} V$. Since it is also completely reducible, it must be isomorphic to $V^{\oplus d}$ for some $d \leq n$. Therefore $\operatorname{End}_{\bar{R}} \bar{R}=\operatorname{End}_{R} \bar{R}=M_{d}(K)$. On the other hand $\operatorname{End}_{\bar{R}} \bar{R}=\bar{R}^{\text {opp }}$. It follows that $\bar{R}$ is isomorphic to $M_{d}(K)$. But then, since $\bar{R}$ has an $n$-dimensional simple module (the module $V$ ), $d$ must equal $n$.

\section{TENSOR PRODUCTS}

The simplest way to understand tensor products is to work with bases. If $V$ and $W$ are vector spaces with bases $x_{1}, \ldots, x_{n}$ and $y_{1}, \ldots, y_{m}$ respectively, their tensor product is the vector space whose basis is given by formal symbols $x_{i} \otimes y_{j}$, and is usually denoted by $V \otimes W$. The map on bases

$$
\left(x_{i}, y_{j}\right) \mapsto x_{i} \otimes y_{j}
$$


extends uniquely to a bilinear map $B: V \times W \rightarrow V \otimes W$, which we shall call the tensor product map. For $(x, y) \in V \times W$, the vector $B(x, y)$ in $V \otimes W$ is usually denoted by $x \otimes y$. In general, we can write

$$
x=a_{1} x_{1}+\cdots+a_{n} x_{n} \text { and } y=b_{1} y_{1}+\cdots+b_{m} y_{m},
$$

for unique scalars $a_{1}, \ldots, a_{n}$ and $b_{1}, \ldots, b_{m}$. Then

$$
x \otimes y=\sum_{i=1}^{n} \sum_{j=1}^{m} a_{i} b_{j}\left(x_{i} \otimes y_{j}\right) .
$$

Starting with different bases of $V$ and $W$ would have led to an apparently different description of the tensor product; let us provisionally denote it by $V \otimes W$. Write $B^{\prime}$ for the corresponding tensor product map $V \times W \rightarrow V \otimes W$. However, the tensor product is independent of the choice of basis in the sense that there exists a unique isomorphism $\phi: V \otimes W \rightarrow V \otimes W$ such that $B^{\prime}=\phi \circ B$. This situation is visually described by asserting that the diagram

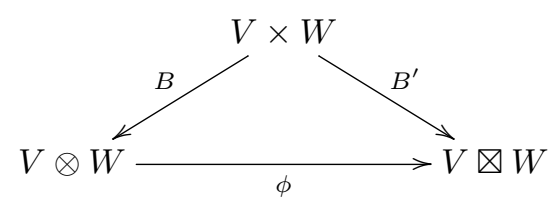

commutes (in other words, it does not matter along which path you compose the functions; the end result is the same). One may try to work out this isomorphism $\phi$ in terms of the change-of-basis matrices.

An interesting alternative is to use the following basis-free characterization of the tensor product [2, Section 8.10]:

Exercise 6.1 (Universal property of tensor products). Show that the tensor product $V \otimes W$ has the following property: For every vector space $U$ and every bilinear map $D: V \times W \rightarrow U$, there exists a unique linear transformation $\tilde{D}: V \otimes W \rightarrow U$ such that $D=\tilde{D} \circ B$. In other words, there exists a unique linear transformation $\tilde{D}$ such that the diagram

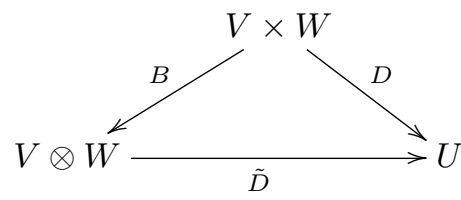

commutes.

Applying the result of Exercise 6.1 to $U=V \otimes W$ and $D=B^{\prime}$, we find that there exists a unique linear transformation $\phi: V \otimes W \rightarrow V \otimes W$ such that the diagram (15) commutes. Exercise 6.1 is also valid with $V \otimes W$ instead of $V \otimes W$. Now taking $U=V \otimes W$ and $D=B$, we obtain a linear transformation $\psi: V \otimes W \rightarrow V \otimes W$ such that

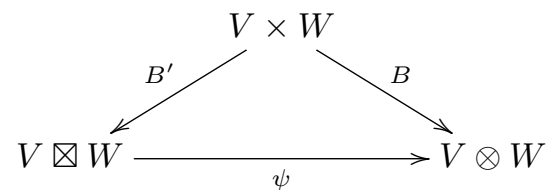

The universal property of tensor products allows for a basis-free characterization. 
commutes. By putting the two diagrams together we get

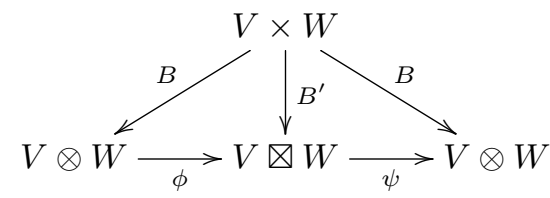

whence it follows that the composition $\psi \circ \phi$ satisfies the commutative diagram

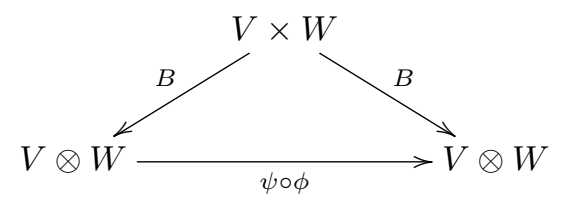

However, this diagram would still commute if we replace $\psi \circ \phi$ by $\operatorname{id}_{V \otimes W}$. The uniqueness assertion in Exercise 6.1 with $U=V \otimes W$ and $D=B$ forces that $\psi \circ \phi=\mathrm{id}_{V \otimes W}$. Similarly, it follows that $\phi \circ \psi=\mathrm{id}_{V \otimes W}$. Thus $\phi$ is an isomorphism.

In the above reasoning, it is does not matter how $V \otimes W$ and $V \otimes W$ are constructed. The conclusion is that any two models of the tensor product which satisfy the universal property of Exercise 6.1 are identified by a unique isomorphism (in this context, a model for the tensor product includes two pieces of informations: the vector space $V \otimes W$ as well as the bilinear map $B: V \times W \rightarrow V \otimes W$ ). Thus all the different models of tensor product are identified with each other via unique isomorphisms.

If $S: V_{1} \rightarrow V_{2}$ and $T: W_{1} \rightarrow W_{2}, B_{i}: V_{i} \times W_{i} \rightarrow V_{i} \otimes W_{i}$ are tensor product maps, since $(x, y) \mapsto B_{2}(S(x), T(y))$ is a bilinear map $V_{1} \times W_{1} \rightarrow V_{2} \otimes W_{2}$, by Exercise 6.1, there exists a unique linear map $S \otimes T: V_{1} \otimes W_{1} \rightarrow V_{2} \otimes W_{2}$ such that

$$
(S \otimes T) \circ B_{1}=B_{2}(S(x), T(y)) .
$$

Classically, $S \otimes T$ is known as the Kronecker product of $S$ and $T$.

Exercise 6.2. Show that $(S, T) \mapsto S \otimes T$ induces an isomorphism $\operatorname{Hom}\left(V_{1}, V_{2}\right) \otimes$ $\operatorname{Hom}\left(W_{1}, W_{2}\right) \rightarrow \operatorname{Hom}\left(V_{1} \otimes V_{2}, W_{1} \otimes W_{2}\right)$.

Exercise 6.3. Show that $\operatorname{trace}(S \otimes T)=(\operatorname{trace} S)(\operatorname{trace} T)$.

Definition 6.4. Suppose $(\rho, V)$ is a representation of $G$ and $(\sigma, W)$ is a representation of $H$. Then $\rho \bigotimes \sigma:(g, h) \mapsto \rho(g) \otimes \sigma(h)$ is a representation of $G \times H$ on $V \otimes W$, which is known as the external tensor product of $(\rho, V)$ and $(\sigma, W)$.

Remark 6.5. The notion of tensor product defined above for representations of two groups is called the external tensor product. There is also a notion of internal tensor product $(\rho \otimes \sigma, V \otimes W)$, which is defined when $(\rho, V)$ and $(\sigma, W)$ are both representations of the same group $G$. This is nothing but the external tensor product (which is a representation of $G \times G$ ) restricted to $\{(g, g) \mid g \in G\}$, the diagonal copy of $G$ inside $G \times G$.

Exercise 6.6. If $V^{\prime}=\operatorname{Hom}_{K}(V, K)$ is the dual vector space of a vector space $V$ then for any vector space $W$ the linear map $V^{\prime} \otimes W \rightarrow \operatorname{Hom}_{K}(V, W)$ induced by the bilinear map $V^{\prime} \times W \rightarrow \operatorname{Hom}_{K}(V, W)$ defined by

$$
(\xi, y) \mapsto(x \mapsto \xi(x) y)
$$

is an isomorphism of vector spaces. 
Exercise 6.7. Let $(\rho, V)$ and $(\sigma, W)$ be representations of groups $G$ and $H$ respectively. Then $\left(\rho^{\prime} \otimes \sigma, V^{\prime} \otimes W\right)$ is a representation of $G \times H$ by Exercise 6.8. On the other hand $\operatorname{Hom}(V, W)$ (which by Exercise 6.6 is canonically isomorphic to the vector space $\left.V^{\prime} \otimes W\right)$ also a representation of $G \times H$ via $\tau: G \times H \rightarrow \operatorname{GL}(\operatorname{Hom}(V, W))$ defined by

$$
\tau(g, h)(T)=\sigma(h) \circ T \circ \rho(g)^{-1} .
$$

Show that the isomorphism of Exercise 6.6 is in fact an intertwiner of representations of $G \times H$. Thus $V^{\prime} \otimes W$ and $\operatorname{Hom}(V, W)$ are isomorphic representations of $G \times H$.

Exercise 6.8. Assume that $K$ is algebraically closed and that its characteristic divides neither $|G|$ nor $|H|$. Show that if $\rho$ and $\sigma$ are simple then so is $\rho \otimes \sigma$ (hint: use Burnside's theorem (Theorem 5.15) and Exercise 6.6). If, furthermore $(\tau, U)$ and $(\theta, X)$ are simple representations of $G$ and $H$ respectively such that $\rho \otimes \sigma$ is isomorphic to $\tau \otimes \theta$ then $\rho$ is isomorphic to $\tau$ and $\sigma$ is isomorphic to $\theta$.

\section{Characters}

In this section, we make the Wedderburn decomposition explicit when $R=$ $K[G]$. We assume that $K$ is an algebraically closed field whose characteristic does not divide $|G|$. The simple $K[G]$-modules are just the simple representations of $G$ (under the correspondence discussed in Section 1. Each simple representation $(\rho, V)$ corresponds to a matrix algebra in the Wedderburn decomposition of $R$ (Corollary 5.11). The identity matrix in this matrix algebra is a primitive central idempotent of $K[G]$, which we denote by $\epsilon_{\rho}$ (see Exercise 5.13). The element $\epsilon_{\rho}$ can be viewed as a $K$-valued function $g \mapsto \epsilon_{\rho}(g)$ on $G$, as explained in Section 1. Our goal shall be the determination of the values $\epsilon_{\rho}(g)$ in terms of $\rho$ and $g$. The answer (17) highlights the important role that characters play in representation theory.

Definition 7.1 (Contragredient Representation). Let $(\rho, V)$ be a representation of $G$. The contragredient representation is the representation $\left(\rho^{\prime}, V^{\prime}\right)$ where $V^{\prime}$ is the vector space dual to $V\left(V^{\prime}=\operatorname{Hom}_{K}(V, K)\right)$ and $\rho^{\prime}(g)^{-1}$ is the adjoint of the linear operator $\rho(g)$, namely the linear operator for which

$$
\left(\rho^{\prime}(g)^{-1}(\xi)\right)(x)=\xi(\rho(g)(x)) \text { for all } \xi \in V^{\prime} \text { and } x \in V .
$$

If $(\rho, V)$ is a representation of $G$ then $\left(\rho \otimes \rho^{\prime}, V \otimes V^{\prime}\right)$ is a representation of $G \times G$ defined by

$$
\left(\rho \otimes \rho^{\prime}\right)\left(g, g^{\prime}\right)(x \otimes \xi)=[\rho(g) x] \otimes\left[\rho^{\prime}\left(g^{\prime}\right) \xi\right] .
$$

By combining the left and right regular modules, $K[G]$ can also be considered a representation of $G \times G$ :

$$
T\left(g, g^{\prime}\right) 1_{x}=1_{g^{\prime} x g^{-1}} .
$$

These two representations of $G \times G$ are linked by matrix coefficients:

Lemma 7.2. Let $c: V \otimes V^{\prime} \rightarrow K[G]$ be defined by

$$
c(x \otimes \xi)=\sum_{g \in G} \xi(\rho(g) x) 1_{g} .
$$

Then $c$ is an intertwiner of representations of $G \times G$. 


\section{Matrix coefficients}

Suppose $x_{1}, \ldots, x_{n}$ is a basis of $V$ and $\xi_{1}, \ldots, \xi_{n}$ is the dual basis of $V^{\prime}$ (the linear functional $\xi_{i}$ is defined by $\left.\xi_{i}\left(x_{j}\right)=\delta_{i j}\right)$. Then $\xi_{i}\left(\rho(g) x_{j}\right)$ is the $(i, j)$ th entry of the matrix of $\rho(g)$ with respect to the basis $x_{1}, \ldots, x_{n}$. Given $(x, \xi) \in$ $V \times V^{\prime}$ it is always possible to find a basis $x_{1}, \ldots, x_{n}$ such that $x=x_{1}$ and $\xi=\xi_{1}$. For this reason $\xi(\rho(g) x)$ is called a matrix coefficient of $\rho(g)$.

A generalization of Theorem 7.3 to compact topological groups is called the Peter-Weyl theorem.

Theorem 7.3. Let $\left(\rho_{1}, V_{1}\right),\left(\rho_{2}, V_{2}\right), \ldots,\left(\rho_{k}, V_{k}\right)$ be a set of representatives for the isomorphism classes of simple representations of $G$. The linear map

$$
\Phi: \bigoplus_{i=1}^{k} V_{i} \otimes V_{i}^{\prime} \rightarrow K[G]
$$

defined by

$$
x_{i} \otimes \xi_{i} \mapsto c\left(x_{i} \otimes \xi_{i}\right) \text { for } x_{i} \in V_{i}, \xi_{i} \in V_{i}^{\prime}
$$

is an isomorphism of representations of $G \times G$.

Proof. Note that $\Phi$ is an intertwiner of representations of $G \times G$ by Lemma 7.2. Since $\Phi$ is a linear transformation between two vector spaces which have (by the Wedderburn decomposition) the same dimension, it suffices to show that $\Phi$ is injective.

By Exercise 6.8, the $V_{i}^{\prime} \otimes V_{i}$ are pairwise non-isomorphic simple representations of $G \times G$. By Exercise 3.15, the kernel of $\Phi$, being an invariant subspace, must be a sum of some subcollection of the $V_{i}^{\prime} \otimes V_{i}$. However, none of the subspaces $V_{i}^{\prime} \otimes V_{i}$ can be contained in the kernel, since for any non-zero vector $v_{i} \in V_{i}$, there exists $\xi_{i} \in V_{i}^{\prime}$ such that $\Phi\left(\xi_{i} \otimes v_{i}\right)(1)=\xi_{i}\left(v_{i}\right) \neq 0$.

Exercise 7.4. Work out the isomorphism of Theorem 7.3 for $G=\mathbf{Z} / 3 \mathbf{Z}$. What about other cyclic groups?

If we restrict the representation $K[G]$ of $G \times G$ to the second copy of $G$ in $G \times G$, we get the left regular representation of $G$. On the other hand, the restriction of the representation $V \otimes V^{\prime}$ of $G \times G$ to the second copy of $G$ is isomorphic to $V^{\prime \oplus \operatorname{dim} V}$. Theorem 7.3 therefore gives a decomposition of the regular representation:

Theorem 7.5. In the notation of Theorem 7.3, the left regular represenation of $G$ has decomposition

$$
K[G] \cong \bigoplus_{i=1}^{k} V_{i}^{\oplus \operatorname{dim} V_{i}},
$$

so each simple representation of $G$ occurs in the regular representation with multiplicity equal to its dimension.

Applying the result of Exercise 6.7 to the case where $(\sigma, W)=(\rho, V)$, we find that $V \otimes V^{\prime}$ and $\operatorname{End}(V)$ are isomorphic as representations of $G \times G$. Under this isomorphism of $V \otimes V^{\prime}$ with linear endomorphisms of $V$, matrix coefficients correspond to trace:

The natural pairing on $V^{\prime} \times V$ becomes the trace of the associated rank-one operator.
Theorem 7.6. Let $V$ be a finite dimensional vector space and $V^{\prime}$ be its dual. Then for every $(\xi, y) \in V^{\prime} \times V, \xi(y)$ is the trace of the linear operator

$$
T_{\xi, y}: x \mapsto \xi(x) y .
$$


Proof. Extend $y$ to a basis of $V$. The matrix of $T_{\xi, y}$ with respect to any such basis has only one non-zero column, namely the first one. Its trace is therefore the element in the first row and first column, which is $\xi(y)$.

Theorem 7.6 can be restated by saying that the following diagram commutes:

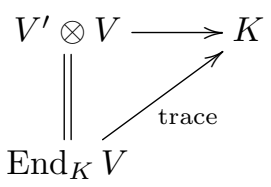

where the horizontal arrow represents the linear map $V^{\prime} \otimes V \rightarrow K$ induced by the bilinear map $(\xi, x) \mapsto \xi(x)$ and the vertical equality represents the canonical linear isomorphism $V^{\prime} \otimes V \rightarrow \operatorname{End}_{K} V$.

Using the fact that $\operatorname{End}_{K} V$ is isomorphic to $V^{\prime} \otimes V$ as a representation of $G \times G$, we may rewrite Theorem 7.3 as

Theorem 7.7. Let $\left(\rho_{1}, V_{1}\right),\left(\rho_{2}, V_{2}\right), \ldots,\left(\rho_{k}, V_{k}\right)$ be a set of representatives for the isomorphism classes of simple representations of $G$. The linear map

$$
\Phi: \bigoplus_{i=1}^{k} \operatorname{End}_{K}\left(V_{i}\right) \rightarrow K[G]
$$

defined by

$$
T_{i} \mapsto \sum_{g \in G} \operatorname{trace}\left(\rho(g) T_{i}\right) 1_{g} \text { for } T_{i} \in \operatorname{End}_{K}\left(V_{i}\right)
$$

is an isomorphism of representations of $G \times G$.

Exercise 7.8. Show that $\operatorname{End}_{G \times G} K[G]$ is the centre of $K[G]$ (you may use Theorem 5.4 as a starting point for this).

It follows (using Exercise 3.12, or independently from Theorem 7.3 and Exercise 6.8 ) that $K[G]$ has a multiplicity-free decomposition into simple representations of $G \times G$. Both, the Wedderburn decomposition (the version given in Corollary 5.12) and Theorem 7.7 are decompositions of $K[G]$ into a sum of simple representations of $G \times G$. Therefore, they must coincide on each simple factor up to a scalar. It follows that for each of the primitive central idempotent $\epsilon_{i}$, which is the image of the identity element of $\operatorname{End}_{K}\left(V_{i}\right)$ is given by

$$
\epsilon_{i}(g)=c_{i} \operatorname{trace}(\rho(g))
$$

where $c_{i}$ is some constant depending on $i$.

Note that if $V$ and $W$ are two finite dimensional vector spaces over $K, T \in$ $\operatorname{End}_{K}(V)$ and $S: V \rightarrow W$ is an isomorphism, then $T$ and $S T S^{-1}$ have the same trace. It follows that isomorphic representations have the same character.

This allows us to equate the traces on both side of the isomorphism

$$
K[G] \cong \bigoplus_{i=1}^{r} V_{i}^{\oplus \operatorname{dim} V_{i}}
$$


of Theorem 7.5 to obtain the identity

$$
\begin{aligned}
1_{e} & =\frac{1}{|G|} \sum_{i=1}^{r} \operatorname{dim} V_{i} \sum_{g \in G} \operatorname{trace}\left(\rho_{i}(g)\right) 1_{g} \\
& =\frac{1}{|G|} \sum_{i=1}^{r} \operatorname{dim} V_{i} c_{i}^{-1} \epsilon_{i}
\end{aligned}
$$

The right hand side is a linear combination of linearly independent vectors $\epsilon_{1}, \ldots, \epsilon_{r}$ in the vector space $K[G]$. On the other hand, we already know that

$$
1_{e}=\sum_{i=1}^{r} \epsilon_{i}
$$

It follows immediately that $c_{i}=\operatorname{dim} V_{i} /|G|$, or in other words that

Theorem 7.9. Let $G$ be a finite group and $K$ be an algebraically closed field whose characteristic does not divide $|G|$. Let $\left(\rho_{1}, V_{1}\right), \ldots,\left(\rho_{r}, V_{r}\right)$ be a set of representa-

The primitive central idempotents are determined by the characters of simple representations. tives for the isomorphism classes of simple representations of $G$ over the field $K$. Let

$$
\epsilon_{i}(g)=\frac{\operatorname{dim} V_{i}}{|G|} \operatorname{trace}\left(\rho_{i}(g)\right)
$$

Then $\epsilon_{1}, \ldots, \epsilon_{r}$ are the primitive central idempotents in $K[G]$.

Definition 7.10 (Character). If $G$ is a group and $\rho: G \rightarrow \mathrm{GL}(V)$ is a representation of $G$ on a finite dimensional vector space $V$ then the $K$-valued function

$$
\chi_{\rho}(g)=\operatorname{trace}(\rho(g))
$$

is called the character of $\rho$.

Exercise 7.11. If $\chi$ is the character of a representation in a complex vector space, then $|\chi(g)| \leq \chi(1)$ for all $g \in G$. Equality holds if and only if $g$ lies in kernel of the corresponding representation. Hint: show that $\chi(g)$ is the trace of a matrix whose eigenvalues are unit complex numbers.

If $\left(\rho_{1}, V_{1}\right), \ldots,\left(\rho_{k}, V_{k}\right)$ are a set of representatives for the isomorphism classes of simple representation of $G$, and we write $\chi_{i}(g)$ for $\operatorname{trace}\left(\rho_{i}(g) ; V_{i}\right)$ then the functions $\chi_{1}, \ldots, \chi_{r}$ are called the irreducible characters of $G$. An immediate consequence of Theorem 7.9 is that $\left\{\chi_{1}, \ldots, \chi_{r}\right\}$ is a linearly independent set in $K[G]$. Now suppose that a representation $(\rho, V)$ has a decomposition into simples given by

$$
V_{1}^{\oplus m_{1}} \oplus \cdots \oplus V_{r}^{\oplus m_{r}}
$$

then

$$
\chi_{\rho}=m_{1} \chi_{1}+\cdots+m_{r} \chi_{r} .
$$

By the linear independence of irreducible characters, the coefficients $m_{1}, \ldots, m_{r}$ and hence the isomorphism class of $(\rho, V)$ is completely determined by $\chi_{\rho}$. We have

Theorem 7.12. Let $K$ be an algebraically closed field whose characteristic does not divide $|G|$. If two finite dimensional representations of $G$ have the same character, then they are isomorphic.

Their most important properties follow from the characterization (17) of primitive central idempotents: 
Exercise 7.13 (Schur's orthogonality relations). When $K$ is an algebraically closed field whose characteristic does not divide $|G|$, then the irreducible characters $\chi_{1}, \ldots, \chi_{r}$ form a basis for the centre of $K[G]$ (which is the space of $K$-valued functions on $G$ which are constant on conjugacy classes, as we have seen in the proof of Corollary 5.14) and satisfy the identities

$$
\frac{1}{|G|} \sum_{g \in G} \chi_{i}(g) \chi_{j}\left(g^{-1} h\right)=\delta_{i j} \frac{\chi_{i}(h)}{\chi_{i}(1)}
$$

which, upon substituting $h=1$, give Schur's orthogonality relations:

$$
\frac{1}{|G|} \sum_{g \in G} \chi_{i}(g) \chi_{j}\left(g^{-1}\right)=\delta_{i j}
$$

In other words, if we define a bilinear map $K[G] \times K[G] \rightarrow K$ by

$$
\left\langle f_{1}, f_{2}\right\rangle_{G}=\frac{1}{|G|} \sum_{g \in G} f_{1}(g) f_{2}\left(g^{-1}\right),
$$

then the irreducible characters form an orthonormal set for this pairing.

An obvious consequence of Theorem 18 is that when the characteristic of $K$ does not divide $|G|$, then the number of isomorphism classes of simple representations is the number of conjugacy classes in $G$. Further, each irreducible character of $G$ is a function on the conjugacy classes. The character table of $G$ is the square array whose rows are indexed by the isomorphism classes of the simple representations of $G$ and whose columns are indexed by its conjugacy classes. Each entry is the value of the irreducible character of the simple representation indexing its row evaluated at any element of the the conjugacy class indexing its column.

The importance of the identities (18) is that they allow us to calculate the dimensions of intertwiner spaces between representations (and hence also multiplicities of the simple representation in a given representation) using characters.

Theorem 7.14. Let $K$ be an algebraically closed field whose characteristic does not divide $|G|$. Let $(\rho, V)$ and $(\sigma, W)$ be two finite dimensional representations of $G$ over $K$. Then

$$
\operatorname{dim} \operatorname{Hom}_{G}(V, W)=\left\langle\chi_{\rho}, \chi_{\sigma}\right\rangle_{G} .
$$

Proof. Suppose that $V_{1}, \ldots, V_{r}$ is a set of representatives for the isomorphism classes of simple representations of $G$. If $V$ and $W$ have decompositions given by equations (6) and (7) respectively, then

$$
\begin{gathered}
\chi_{\rho}=n_{1} \chi_{1}+\cdots+n_{r} \chi_{r} \\
\chi_{\sigma}=m_{1} \chi_{1}+\cdots+m_{r} \chi_{r} .
\end{gathered}
$$

Now using the fact that $\chi_{1}, \ldots, \chi_{r}$ form an orthonormal set for the pairing (19) we have that

$$
\left\langle\chi_{\rho}, \chi_{\sigma}\right\rangle=m_{1} n_{1}+\cdots+m_{r} n_{r},
$$

which, by Theorem 3.5, is the dimension of $\operatorname{Hom}_{G}(V, W)$.

As a special case, we may compute the multiplicity of a simple representation in any given representation using characters: 
Theorem 7.15. Let $K$ be an algebraically closed field whose characteristic does not divide $|G|$. Suppose that a simple representation $(\rho, V)$ of $G$ occurs in a representation $(\sigma, W)$ with multiplicity $m$. Then

$$
m=\left\langle\chi_{\rho}, \chi_{\sigma}\right\rangle_{G} .
$$

Exercise 7.16. Compute the character table for the following groups:

(1) finite cyclic groups

(2) (2) the dihedral group of order 8 (this is the group of symmetries of a square)

(3) (5) the quaternion group (this is the group consisting of the elements $\{ \pm 1, \pm \mathbf{i}, \pm \mathbf{j}, \pm \mathbf{k}\}$ in the ring of quaternions.

\section{Dihedral Groups}

The dihedral group $D_{2 n}$ is defined to be the group of linear transformations $\mathbf{R}^{2} \rightarrow \mathbf{R}^{2}$ which fixes the vertices of a regular $n$-gon centred at the origin. This group consists of rotations (by angles that are multiples of $2 \pi / n$ ) and reflections about the $n$ axes of symmetry of the $n$-gon, and therefore has order $2 n$. If $s$ denotes any one of the reflections, and $r$ a rotation by $2 \pi / n$, then $D_{2 n}$ has a presentation

$$
\left\langle s, r \mid s^{2}=1, r^{n}=1, s^{-1} r s=r^{-1}\right\rangle
$$

Exercise 7.17. Let $g_{1}, g_{2}, \ldots, g_{r}$ denote representatives of the conjugacy classes of $G$. Let $\chi_{1}, \chi_{2}, \ldots, \chi_{r}$ be the characters of the simple representations of $G$. Ley $X$ denote the matrix whose $(i, j)$ th element is $\chi_{i}\left(g_{j}\right)$. Let $Z$ denote the diagonal matrix whose $i$ th diagonal entry is the cardinality of the centralizer of $g_{i}$ for each $i \in\{1, \ldots, r\}$. Let $E$ denote the permutation matrix for which $E_{i j}=1$ if $g_{i}^{-1}$ lies in the conjugacy class of $g_{j}$. When $K$ is an algebraically closed field whose characteristic does not divide $|G|$, show that $X^{\prime} X=E Z$ (here $X^{\prime}$ denotes the transpose of $X$ ). Use this to deduce the dual orthogonality relations:

$$
\sum_{k=1}^{r} \chi_{k}\left(g_{i}^{-1}\right) \chi_{k}\left(g_{j}\right)= \begin{cases}\left|Z_{G}\left(g_{j}\right)\right| & \text { if } i=j, \\ 0 & \text { otherwise. }\end{cases}
$$

[Hint: start off by computing the matrix $X X^{\prime}$.]

Exercise 7.18. When $K$ is an algebraically closed field whose characteristic does not divide $|G|$, show that thet centre $Z(G)$ of $G$ consists of those elements for which $|\chi(g)|=\chi(1)$ for each irreducible character $\chi$.

\section{REFERENCES}

[1] N. Jacobson, Basic Algebra I, Dover 2009.

[2] G. Birkhoff and S. Mac Lane, A survey of Modern Algebra, A. K. Peters, 2008. 